\title{
Caricature, satire et parodie : l'affranchissement des lois du genre
}

Gaël HENAFF

Maître de conférences, HDR, université de Rennes 2

\section{Citation}

Gaël Henaff, "Caricature, satire et parodie : l'affranchissement des lois du genre" in Défier la décence. Crise du sens et nouveaux visages du scandale dans l'art, L. Brogowski, J. Delaplace et J. Laurent (dir.),, Artois Presses Université, 2016, p.185 s.

En septembre 2012, les nouvelles publications par Charlie Hebdo de caricatures prenant pour cible les religions ont relancé, au moins pour un temps, les débats sur les limites de la caricature et de la provocation. L'année 2011 avait été également riche en événements : incendie des locaux de Charlie hebdo après la parution d'un numéro retitré Charia Hebdo, manifestations intégristes contre la pièce Golgota Picnic de l'Argentin Rodrigo Garcia ou encore interruption de la représentation de la pièce Sur le concept du visage du fils de Dieu de Romeo Castellucci.

Jusqu'où le caricaturiste peut-il aller ? Quelles sont les limites à ne pas dépasser dans l'usage de la dérision ? Où s'arrête la liberté d'expression et où commence l'abus ? C'est à ces questions que les juges apportent peu à peu des réponses, au gré des actions en justice engagées par des victimes qui manifestent en tout cas que le provocateur a dépassé le seuil de leur tolérance.

La règle de référence serait la « loi du genre », que l'on trouve mentionnée comme exception aux droits d'auteur ou aux droits voisins dans le code de la propriété intellectuelle (respectivement CPI art. L122-5 $4^{\circ}$ et L211-3 $4^{\circ}$ ). L'auteur d'une œuvre de l'esprit ne peut interdire « la parodie, le pastiche et la caricature, compte tenu des lois du genre ».

Suggérons, après d'autres ${ }^{1}$, que les lois du genre renvoient aux usages, « hommage » ou simple renvoi de la loi aux règles de l'art. Ces usages varieraient selon le «genre » satirique considéré. La caricature aurait ses règles qui ne seraient pas celles du pastiche, pas plus que de la parodie et réciproquement. Ces genres répondent chacun à des règles que l'expert serait habile à distinguer même si le public les confond bien volontiers. La parodie porterait sur une œuvre, transformée de façon comique, ludique ou satirique. Son but est «d'attirer

\footnotetext{
${ }^{1}$ Pierre-Yves Gautier, Propriété littéraire et artistique, Paris, PUF, 2004 ; Christophe Caron, Droit d'auteur et droits voisins, Paris, Litec, 2009 ; en ce sens Michel Vivant, Jean-Michel Bruguière, Droits d'auteur et droits voisins, Paris, Dalloz, 2013.
} 
l'attention du lecteur ou du spectateur par un alliage de familier et de nouveau, de provoquer chez lui le double plaisir de la reconnaissance et de la surprise ${ }^{2} \gg$. Le pastiche, qui se distingue en cela de la parodie avec laquelle il est souvent confondu, aurait pour objet «l'imitation stylistique ». La caricature part d'une situation réelle, d'une personne, dont les traits sont exagérés à un point tel qu'elle devient ridicule. Elle se manifeste par le «portrait en charge, qui utilise la déformation physique comme métaphore d'une idée (portrait politique) ou se limite à l'exagération des caractères physiques (portraits d'artistes) » et par « la caricature de situation, dans laquelle des événements réels ou imaginaires mettent en relief les mœurs ou le comportement de certains groupes humains ${ }^{3} »$.

Ces distinctions ne reçoivent pourtant aucun écho en droit positif, en doctrine, comme en jurisprudence. On lit plutôt que la parodie concernerait les œuvres musicales, le pastiche les œuvres littéraires et la caricature les œuvres d'art graphiques et plastiques ${ }^{4}$. Mais cette distinction n'est pas vraiment convaincante. Le détournement ne se laisse pas facilement enfermer dans un genre unique ${ }^{5}$. D'ailleurs, les auteurs s'accordent à considérer qu'il s'agit là d'une distinction «technique »sans conséquence juridique ${ }^{6}$. La jurisprudence, qui a semblé un temps juger qu'il est « dans les lois du genre » de la parodie «de permettre l'identification immédiate de l'œuvre parodiée » tandis que le pastiche consiste à « se moquer d'un personnage par l'intermédiaire de l'œuvre caricaturée dont il est $l^{\prime}$ auteur ${ }^{7} \gg$ semble également renoncer à toute distinction.

Les lois du genre ne sont pas tant forgées par la pratique des parodistes que par une autre habitude, la jurisprudence. C'est ce qu'il ressort des analyses fondées sur de très nombreuses décisions de justice mettant en évidence deux éléments caractéristiques de la satire : une intention humoristique et l'absence de confusion entre l'œuvre parodique et l'œuvre parodiée ${ }^{8}$.

\footnotetext{
${ }^{2}$ Daniel Sangsue, « Parodie », in Encyclopedia universalis.

${ }^{3}$ Marc Thivolet, « Caricature», in Encyclopedia universalis.

${ }^{4}$ La distinction, attribuée à Henri Desbois, Le Droit d'auteur en France, Paris, Dalloz, 1978, p. 323, est encore fréquemment reprise.

${ }^{5}$ Marik Froidefond, «De l'ostinato au pastiche : approche comparée du comique de répétition en poésie et en musique au $\mathrm{XX}^{\mathrm{e}}$ siècle », Études littéraires, vol. 38, n²-3, 2007, p. 71 et sv.; Umberto Eco, Pastiches et Postiches, Paris, Grasset, 1992.

${ }^{6}$ Caron, op. cit. ; Gautier, op. cit. ; André Lucas, Propriété littéraire et artistique, Paris, Dalloz, 2010, p. 66 ; Vivant, Bruguière, Droits d'auteur et droits voisins, op. cit.

${ }^{7}$ Civ. $1^{\text {re }}, 12$ janv. $1988, n^{\circ} 85-18787$ : Bull. civ. I, ${ }^{\circ} 5$.

${ }^{8}$ Frédérique Fiechter-Boulvard, «La caricature : dualité ou unité ?», Recueil Dalloz 1997, p. 67 ; André Françon obs. sous civ. $1^{\text {re }} 12$ janv. 1988 : RIDA 1988, p. 98 et obs. sous TGI Paris, 7 oct. 1992 : RTD Com. 1993, p. 96 ; Vivant, Bruguière, Droits d'auteur et droits voisins, op. cit. ; André R. Bertrand, Droit d'auteur, Paris, Dalloz, 2010 ; Caron, op. cit.
} 
L'intention humoristique est souvent présentée comme l'élément intentionnel caractéristique de la caricature ou de la parodie. Mais quelles sont les frontières du comique ${ }^{9}$ dans un domaine où «l'excès est la loi du genre ${ }^{10}{ }$ ? La jurisprudence est souvent difficile à déchiffrer, et pour cause : «quand le principal instrument de mesure de celle-ci est aussi variable et propre à chaque individu que l'humour, l'insécurité juridique devient extrême ${ }^{11}$ ».

L'autre condition, l'élément matériel de la caricature, tiendrait dans l'évidence de l'imposture. Le public ne doit pas confondre la parodie avec la réalité, l'œuvre parodiée ou le personnage caricaturé ${ }^{12}$. L'élément matériel découle bien souvent de l'élément intentionnel dès lors que la caricature vise à faire rire à partir d'un modèle d'origine sérieux. Dans le cas contraire, l'hésitation est permise ${ }^{13}$. Cette exigence risque de conduire l'auteur du pastiche à de grossières réalisations atteignant ainsi les limites du genre ${ }^{14}$. L'exercice est décidément bien périlleux.

La plupart des décisions de justice rattachent bien volontiers la caricature à un genre plus noble, la liberté d'expression, droit essentiel dans une société démocratique comme le rappelle fréquemment la Cour européenne des droits de l'homme et «condition primordiale de son progrès et de son épanouissement ${ }^{15} \gg$. La caricature, souvent provocatrice, y trouvera sa place dès lors qu'il est admis que cette liberté «vaut non seulement pour les "informations" ou "idées" accueillies avec faveur ou considérées comme inoffensives ou indifférentes, mais aussi pour celles qui heurtent, choquent ou inquiètent l'État ou une fraction quelconque de la population $^{16}{ }$. Pourtant, malgré ce rattachement, la caricature peine à se démarquer des lois du genre subissant la double contrainte des limites imprécises de ces lois et de celles relatives à la liberté d'expression : « chacun a le droit de s'opposer à la reproduction de son image et cette reproduction, sous forme de caricature, n'est licite, selon les lois du genre, que pour assurer le plein exercice de la liberté d'expression ${ }^{17}{ }$.

\footnotetext{
${ }^{9}$ Gautier, op. cit.; Véronique Staeffen. obs. sous Paris, 4e ch., sect. B, 7 mai 2004 : société Ferrero France : Gaz. Pal. 10 mai 2007, n¹30, p. 12.

${ }^{10}$ TGI Paris, 9 janv. 1992 : Recueil Dalloz 1994, Somm. 195, obs. Christophe Bigot ; dans le même sens, Bernard Edelman, «La Cour de cassation ne rit pas »: Recueil Dalloz 1997, p. 411 ; « Telle est la mission de la parodie : elle ne doit pas craindre d'exagérer ». Eco, op. cit. p. 14.

${ }^{11}$ Emmanuel Baud et Stéphane Colombet, «La parodie de marque : vers une érosion du caractère absolu des signes distinctifs ? », Recueil Dalloz, 1998, p. 227.

${ }^{12}$ Ass. Plen. 12 juill. 2000, $\mathrm{n}^{\circ} 99-19004$ et 99-19.005, « Guignols de l'info »: Bull. AP, n7.

${ }^{13}$ Caricature des feuilles mortes, hommage à Yves Montand par Jacques Faizant, condamnée en première instance, TGI Paris 7 oct. 1992, RTD Com. 1993, p. 96 obs. André Françon, mais validée par Paris, 11 mai 1993 : RTD Com. 1993 p. 510 obs. André Françon.

${ }_{15}^{14}$ Béatrice Martinet obs. sous Paris (4 ch. B), 17 janvier 2003 : Sté Les Jalons : Gaz. Pal. 2003, n³29, p. 24.

${ }^{15}$ Parmi les arrêts fondateurs régulièrement cité, CEDH, Handyside c/ Royaume Uni, 7 déc. 1976, §.49.

${ }^{16}$ Pour juger cependant que l'interdiction de l'ouvrage n'était pas une violation de l'article 10 dans l'affaire Handyside c/ Royaume Uni ; pas plus que la confiscation des toiles jugées obscènes par les autorités suisses dans l'affaire Muller et autres c/ Suisse, 24 mai 1988.

${ }^{17}$ Civ. 1re, 13 janv. 1998, Bull. civ. I, n¹4.
} 
Un certain nombre de contentieux dans des domaines aussi différents que le droit des marques, la liberté de croyance ou les droits de la personnalité atteste pourtant d'une évolution jurisprudentielle qui tend à rattacher la caricature au principe plus général de liberté d'expression en l'affranchissant des lois du genre. Si cette tendance était confirmée, la perspective en serait totalement changée et la caricature deviendrait une liberté et non une simple exception aux droits d'autrui.

\section{La caricature militante et la parodie de marque}

Le détournement d'un signe distinctif, marque ou nom commercial est un genre assez périlleux car, contrairement aux règles applicables aux œuvres de l'esprit, la caricature ou la parodie ne figurent pas explicitement parmi les exceptions au droit des marques. À partir de ce constat, un premier courant d'opinion considère que la protection de la marque est absolue et ne souffre aucune exception. On ne peut transposer l'exception de caricature du droit d'auteur au droit des marques ${ }^{18}$. Un deuxième courant d'opinion défend la suprématie des droits d'auteurs et de ses exceptions, sur les droits de propriété intellectuelle. Les limites aux droits d'auteur s'appliquent aussi bien aux droits voisins qu'au droit des marques ${ }^{19}$. Un troisième courant, intermédiaire, appelle enfin à distinguer entre une parodie de marque dont la finalité est d'inciter à acheter la marque parodiée, et celle qui vise «gratuitement, à procurer un effet comique largement entendu ». Pour être licite, la parodie de marque devrait respecter les conditions de la parodie des œuvres littéraires et artistiques, et, « en plus, ne pas être réalisée à des fins purement commerciales ${ }^{20} »$.

Après bien des péripéties judiciaires dans les affaires ayant opposé les guignols de l'info et l'entreprise PSA et son dirigeant d'alors, c'est encore une autre voie que suivit l'Assemblée Plénière de la Cour de cassation. La caricature est licite dans la mesure où « les propos incriminés relevaient de la liberté d'expression sans créer aucun risque de confusion entre la réalité et l'œuvre satirique ${ }^{21} »$. Toute source de contentieux n'était pas tarie pour autant comme l'illustrent deux affaires opposant l'association Greenpeace à des entreprises commerciales, même en se situant clairement hors de la vie des affaires.

\section{Esso contre Greenpeace et réciproquement}

\footnotetext{
${ }^{18}$ Par ex. Caron, op. cit., n³82 et la jurisprudence citée; Pascale Tréfigny-Goy, note sous Rennes, $2^{\mathrm{e}}$ ch., 27 avr. 2010 , Petit navire : PI 2011, comm. 2, n ${ }^{\circ}$.

${ }^{19}$ Bernard Edelman, Droits d'auteur, droits voisins, Paris, Dalloz, 1993, nº205 ; Bertrand, op. cit., $\mathrm{n}^{\circ} 111.50$.

${ }^{20}$ Baud et Colombet, op. cit., p. 227.

${ }^{21}$ AP 12 juill. 2000, nº9-19004 : préc.
} 
Dans la première série de décisions, l'association Greenpeace était opposée à la société Esso dont elle avait, dans le cadre d'une campagne militante, détourné la marque transformée pour l'occasion en « $\mathrm{E} \$ \$ \mathrm{O}$ » ou «Stop E\$ $\$ \mathrm{O}$ ». En référé, le premier juge avait interdit l'usage de la marque, décision infirmée en appel ${ }^{22}$. L'action ayant été engagée au fond, dans un jugement en date du 30 janvier 2004, le TGI de Paris déboute la société de ses demandes en reprenant les motivations des juges des référés. « Si le droit des marques ignore l'exception de parodie, en revanche le principe à valeur constitutionnelle de la liberté d'expression implique que l'association Greenpeace France puisse (...) dénoncer sous la forme qu'elle estime appropriée au but poursuivi les atteintes portées à l'environnement et les risques causés à la santé humaine par certaines activités industrielles ; que si cette liberté n'est pas absolue, elle ne peut néanmoins subir que les restrictions rendues nécessaires par le respect des droits d'autrui. $^{23} \gg$ On remarquera au passage la précision que le droit des marques ignore l'exception de parodie pour souligner le rattachement du détournement à la liberté d'expression.

Cette position sera reprise par la Cour d'appel de Paris le 16 novembre 2005 et confirmée par la Cour de cassation :

l'usage d'éléments des marques renommées distinguant les produits et services de la société Esso, sous une forme modifiée résumant ces critiques dans un contexte polémique, constituait un moyen proportionné à l'expression de telles critiques ${ }^{24}$.

La décision de la Cour d'appel sera pourtant censurée au motif qu'elle n'avait pas recherché, comme il lui était demandé, si la reprise de la dénomination Esso dans le code source de son site internet n'était pas constitutive d'une faute. L'affaire n'est donc pas tout à fait terminée sur ce point particulier.

\section{Areva contre Greenpeace, Greenpeace contre Areva}

Parallèlement démarrait une affaire comparable opposant cette fois l'association Greenpeace à la Société des Participations du Commissariat à l'énergie atomique (SPCEA), détentrice de la marque Areva. Celle-ci reprochait à celle-là d'avoir reproduit sur des sites internet la lettre A stylisée de la marque et la dénomination Areva en les associant toutes deux à une tête de mort et au slogan «Stop plutonium - l'arrêt va de soi ». Contrairement à ce qui s'était passé dans l'affaire Esso au même stade, la société est déboutée en référé, le juge s’interrogeant sur

\footnotetext{
${ }^{22}$ TGI Paris, ord. réf., 8 juill. 2002 et Paris, 26 févr. 2003 : décisions très commentées par une doctrine que nous ne pouvons citer dans ce cadre.

${ }^{23}$ TGI Paris, 30 janv. 2004 : Comm. com. électr. 2004, comm. 39 note Christophe Caron.

${ }^{24}$ Com., 8 avr. 2008, no66-10961, SA Esso c/ Assoc. Greenpeace France : Recueil Dalloz 2008, AJ, p.1274, obs. Jeanne Daleau ; RTD civ. 2008, p. 487, obs. Patrice Jourdain ; Comm. com. Électr. 2008, comm. 77 note Christophe Caron.
} 
l'existence d'une contrefaçon hors du « terrain commercial ${ }^{25} »$. Saisi au fond, le TGI de Paris juge également que l'usage de la marque est étranger à la vie des affaires et qu'il n'y a pas de contrefaçon de marque ${ }^{26}$, mais retient que l'équation «Areva = mort » est un dénigrement, un abus de la liberté d'expression qui engage la responsabilité civile de son auteur. L'association est condamnée en appel à verser la somme d'un euro symbolique de dommages et intérêts et à supporter les frais de publication de la décision. Ultime dénouement de cette affaire interminable, cet arrêt sera cassé sans renvoi par la Cour de cassation qui considère que l'association Greenpeace n'a pas abusé de son « droit de libre expression ${ }^{27}$ ».

Cet arrêt, plutôt laconique, renvoie bien entendu, la référence «droit de libre expression » en atteste, à la décision rendue en 2006 dans une affaire qui avait opposé le Comité national contre les maladies respiratoires et la tuberculose (CNMRT) contre un fabricant de tabac dont la marque avait été parodiée et associée également à la mort. «En utilisant des éléments du décor des paquets de cigarettes de marque Camel, à titre d'illustration, sur un mode humoristique, dans des affiches et des timbres diffusés à l'occasion d'une campagne générale de prévention à destination des adolescents, dénonçant les dangers de la consommation du tabac, produit nocif pour la santé, le CNMRT, agissant, conformément à son objet, dans un but de santé publique, par des moyens proportionnés à ce but, n'avait pas abusé de son droit de libre expression ${ }^{28} \gg$.

Le juge ne reconnaît donc pas l'exception de parodie dans « la vie des affaires ${ }^{29}$. En dehors de cette «vie des affaires » et c'est le cas dans les débats d'intérêt général, la parodie de marque se trouve rattachée à la liberté d'expression dont elle n'est que l'une des manifestations possibles. On peut regretter cependant, s'agissant d'une l'exercice d'une liberté, qu'elle doive, tout comme les atteintes à cette liberté, être justifiée et proportionnée au but poursuivi. Ce n'est pas en tout cas le régime qui a été retenu pour les caricatures en matière religieuse.

\section{Blasphème, injure : caricatures et liberté de croyance}

\footnotetext{
${ }^{25}$ TGI Paris, ord. réf., 2 août 2002 confirmée par Paris $14^{\mathrm{e}}$ ch., 26 févr. 2003.

${ }^{26}$ TGI Paris, $3^{\mathrm{e}}$ ch., sect. 2, 9 juill. 2004, Sté Participations du CEA c/ Assoc. Greenpeace France : Comm. com. électr. 2004, comm. 110 note Christophe Caron ; confirmation par Paris, $4^{\mathrm{e}}$ ch., sect. B, 27 nov. 2006 : Comm. com. électr. 2007, comm. 8 note Christophe Caron.

${ }^{27}$ Civ. $1^{\text {re }}, 8$ avr. 2008, $\mathrm{n}^{\circ}$ 07-11251: Recueil Dalloz 2008, AJ, p.1207, obs. Sabrina Lavric ; p. 2402, note Laurent Neyret ; RTD civ. 2008, p. 487, obs. Patrice Jourdain ; Comm. com. Électr. 2008, comm. 77 note Christophe Caron.

${ }^{28}$ Civ. $2^{e}$, 19 oct. 2006, $\mathrm{n}^{\circ}$ 05-13489, CNMRT c/ Sté JT International : Recueil Dalloz 2008, Pan. 247, obs. Yves Picod ; Christophe Geiger, «Droit des marques et liberté d'expression, de la proportionnalité de la libre critique »: Recueil Dalloz 2007, Chron. 884 ; JCP 2006. II. 10195, note Frédéric Pollaud-Dullian ; LPA 2008, n 188 , p. 22, note Dorothée GuérinSeysen.

${ }^{29}$ Sur cette notion José Monteiro et Vincent Ruzek, «L'usage du signe des fins autres que celle de distinguer les produits et services d'un opérateur économique » : PI 2007, étude 9. Par ex. Rennes, $2^{\mathrm{e}}$ ch., 27 avr. 2010 : PI 2011, comm. 2 obs. Pascale Tréfigny-Goy.
} 
La liberté de croyance et de conviction, for intérieur, doit être conciliée dans une démocratie avec la liberté d'expression. Cette conciliation nécessaire fut rappelée en 1990 par la cour de cassation à propos du film de M. Scorsese La dernière tentation du Christ (The Last Temptation of Christ) dont on sait que la diffusion lors de sa sortie en France en 1988 avait été accompagnée de plusieurs attentats. La cour d'appel avait considéré « qu'il y avait lieu d'éviter que quiconque se trouve, parce que non prévenu, en situation d'être atteint dans ses convictions profondes » et jugé en l'espèce «qu'en l'absence de trouble manifestement illicite, il ne convenait pas de donner suite à la demande d'interdiction de diffusion et de saisie ». La Cour de cassation rejeta le pourvoi contre cette décision au motif que

le principe de la liberté d'expression, notamment en matière de création artistique, d'une part, comme, d'autre part, celui du respect dû aux croyances et le droit de pratiquer sa religion étant d'égale valeur, il appartenait aux juges du fait de décider des mesures appropriées à faire respecter ce nécessaire équilibre ${ }^{30}$.

C'est sur fond de caricature, et dans l'établissement de principes directeurs de cette conciliation entre liberté de croyance et liberté d'expression ${ }^{31}$, que la Cour européenne des droits de l'homme a pu se prononcer dans l'affaire Otto Preminger Institut du 20 septembre 1994. La Cour européenne reconnaît aux États et aux juges internes une large marge de liberté pour apprécier l'existence et l'étendue de la nécessité d'une limitation de la liberté d'expression, les autorités nationales étant «mieux placées que le juge international » pour évaluer la nécessité de mesures de restriction à la liberté d'expression «à la lumière de la situation qui existe au plan local à une époque donnée ». C'est en application de ces principes que la saisie et la confiscation du film le Concile de l'Amour (Das Liebeskonzil) par les autorités autrichiennes afin de protéger la paix religieuse du Tyrol n'a pas été jugée contraire à l'article 10 de la Convention européenne de sauvegarde des droits de l'homme et des libertés fondamentales $^{32}$. De même, dans l'affaire Wingrove ${ }^{33}$, la saisie par les autorités britanniques du film Vision d'extase (Visions of Ecstasy), court métrage de 18 minutes s'inspirant de la vie et des écrits de sainte Thérèse d'Avila, était jugée conforme à l'article $10^{34}$.

En droit français, les caricatures ou parodies visant les religions ne sont soumises à aucune loi du genre comparable à celle existant en droits d'auteur. La caricature sera donc appréciée à l'aune de la liberté d'expression et de ses abus. Toutefois, ses limites se trouvent

\footnotetext{
${ }^{30}$ Civ. 1re, 29 oct. 1990, n88-19366 : Bull. civ. I, n²26 ; avec les précédents « Je vous salue Marie » : TGI Paris, 28 janv. 1985, AGRIF c/ Godard et Larry Flint : TGI Paris, 20 févr. 1997, Maupeou d’Ableiges c/ Sté Columbia Tristar Films France SA.

${ }^{31}$ Amandine Capitani et Marcel Moritz, « La liberté de caricature et ses limites en matière religieuse », LDI 2006, p. 75 et s.

${ }^{32}$ Par 6 voix contre 3 . Voir les opinions dissidentes et communes de trois juges.

${ }^{33}$ CEDH, Wingrove c/ Royaume Uni, 25 nov.1996, n $17419 / 90$.

${ }^{34}$ Par 7 voix contre 2.
} 
désormais dans la loi sur la presse du 29 juillet 1881 à travers le délit d'injure contre des personnes ou un groupe de personnes à raison «de leur origine ou de leur appartenance ou de leur non appartenance à une ethnie, une nation, une race ou une religion déterminée » (L 29 juill. 1881, art. 29 et 33) ou du délit de provocation à la discrimination et à la haine raciale ou religieuse, que la provocation ait été, ou non, suivie d'effet (L. 29 juill. 1881, art. 24 al. 6). L'existence de ces textes spécifiques exclut en effet que l'on puisse juger au-delà d'abus de la liberté d'expression, comme pour les parodies de marque ${ }^{35}$. Cet affranchissement des lois du genre explique sans doute pour partie certaines décisions plutôt favorables à la parodie. Nous ne prendrons que deux exemples.

\section{Mise en Cène}

Une première affaire concernait une affiche, réalisée pour une marque de vêtements, qui avait été fixée sur la façade d'un immeuble ${ }^{36}$. L'affiche en litige, très «inspirée » de la Cène, célèbre tableau de Léonard de Vinci, représentait les participants du dernier repas du Christ remplacés par des femmes portant des vêtements de la marque et accompagnées d'un homme dos nu.

Les juges du fond retiendront l'injure pour interdire la diffusion. La Cène est «un événement fondateur du christianisme, lors duquel Jésus-Christ institua le sacrement de l'Eucharistie » et fait « incontestablement partie des éléments essentiels de la foi catholique ». «L'installation de l'affiche litigieuse sous la forme d'une bâche géante sur le passage d'un très grand nombre de personnes constitue », selon les magistrats, «l'utilisation dévoyée, à grande échelle, d'un des principaux symboles de la religion catholique, à des fins publicitaires et commerciales. » Le demandeur est bien fondé « à soutenir qu'il est fait gravement injure aux sentiments religieux et à la foi des catholiques. » Le caractère sacré de l'événement détourné, la finalité commerciale de la satire ou la gratuité de l'offense ainsi que sa publicité ont certainement pesé assez lourd dans la décision des juges du fond ${ }^{37}$.

Pourtant, la Cour de cassation censure l'arrêt de la cour d'appel. Revenant à une lecture stricte des dispositions relatives au délit d'injure, elle juge que

\footnotetext{
${ }^{35}$ Ass. plén. 12 juill. 2000, Erulin et Collard, Bulletin AP, $\mathrm{n}^{\circ} 8$; RTD civ. 2000, p. 845 note Patrice Jourdain ; également sur les parodies grossières du journal La Grosse Bertha: Civ 2e, 8 mars 2001, nº98-17574 : Gaz. Pal. 19 juin 2001 n 170, p. 17, avec le rapport de Pierre Guerder et les conclusions de l'avocat général Philippe Chemithe.

${ }^{36}$ Civ. 1re, 14 nov. 2006, n ${ }^{\circ}$ 5-15822, Société GIP contre Association Croyances et libertés et autres : Gaz. Pal. 12 déc. 2006, n³46, p. 9, note Gérard Gonzalez; Gaz. Pal., 6 oct. 2007, n²79, p. 42 obs. Pierre Guerder; Recueil Dalloz, 2007, p.2072 note critique Emmanuel Dreyer.

${ }^{37}$ Voir l'analyse de Patrice Rolland, « La critique, l'outrage et le blasphème » : Recueil Dalloz, 2005, p. 1326.
} 
la seule parodie de la forme donnée à la représentation de la Cène qui n'avait pas pour objectif d'outrager les fidèles de confession catholique, ni de les atteindre dans leur considération en raison de leur obédience, ne constitue pas l'injure, attaque personnelle et directe dirigée contre un groupe de personnes en raison de leur appartenance religieuse.

On peut être étonné que dans l'affaire de la Cène la cour de cassation ait montré tant de tolérance à l'égard d'un détournement publicitaire. La finalité lucrative de l'opération, soulignée à maintes reprises par les juges du fond pour interdire la diffusion de l'affiche, méritaitelle le paravent de la liberté d'expression? Sans aucun doute, même si la protection d'une telle liberté atteint plus rapidement ses limites. La liberté d'expression «ne joue pas seulement pour certains types de renseignements, d'idées ou de modes d'expression [..., elle] englobe aussi les informations à caractère $\operatorname{commercial}^{38} »$.

Les conditions de l'injure, seul fondement possible de la condamnation, n'étaient pas ici réunies ${ }^{39}$. Mais on ne peut s'empêcher de penser que le juge a été conforté dans sa décision par l'origine de l'œuvre et par son esthétique, ainsi que par l'hommage au génie de Léonard de Vinci, dont l'altération de l'œuvre n'était pas discutée. Enfin, le contexte particulier des affaires judiciaires n'est sans doute pas totalement étranger à cette décision. En effet, la Cour de cassation se prononce alors qu'a démarré en France une autre polémique liée aux caricatures de Mahomet.

\section{Charlie c/ Mahomet}

En 2006, Charlie Hebdo publie une série de caricatures de Mahomet qu'un journal danois avait antérieurement publiées, déclenchant de très vives protestations et certaines réactions violentes. Le journal français est poursuivi pour injure envers un groupe de personnes à raison de leur appartenance à une religion déterminée. Trois caricatures sont particulièrement visées. Deux d'entre elles avaient été publiées au Danemark. L'une représentait le prophète accueillant sur son nuage des terroristes avec la légende : «Arrêtez, arrêtez, nous n'avons plus de vierges! ! («Stop, stop, we ran out of virgins »). L'autre le représentait coiffé d'un turban dissimulant une bombe à la mèche allumée, accompagnée de la légende : «Allah est grand et Mahomet est son prophète ». Le journal proposait en couverture une troisième caricature dessinée par Cabu et représentant Mahomet, disant : «C'est dur d'être aimé par des cons ».

\footnotetext{
${ }^{38}$ CEDH, 20 nov. 1989, n¹0572/83, Markt Intern Verlag GmbH et Klaus Beermann, § 26; CEDH 24 févr.1994, $\mathrm{n}^{\circ} 15450 / 89$, Casado Coca c/ Espagne, $\S 35$.

${ }^{39}$ Sur ses aspects techniques, voir E. Dreyer, art. préc.
} 
En mars 2007, le TGI de Paris relaxait les prévenus ${ }^{40}$ en rappelant le principe de liberté d'expression tel qu'exprimé par la Cour européenne des droits de l'homme dans l'arrêt Handyside c/ Royaume Uni du 7 décembre 1976. Le tribunal souligne également que « des restrictions peuvent être apportées à la liberté d'expression si celle-ci se manifeste de façon gratuitement offensante pour autrui, sans contribuer à une quelconque forme de débat public capable de favoriser le progrès dans les affaires du genre humain ».

En l'espèce, le tribunal estime que deux des caricatures ne visent que les intégristes et les terroristes et «n'assimilent pas islam et commission d'actes de terrorisme ». Toute autre est la caricature du prophète à la bombe qui, pour reprendre les termes du jugement « ne porte nullement à rire ou à sourire mais inspire plutôt l'inquiétude et la peur ». Toutefois, si le tribunal juge que ce dessin apparaît, «en soi et pris isolément, de nature à outrager l'ensemble des adeptes de cette foi », c'est pour conclure que dans le contexte (débat sur les caricatures danoises) et les circonstances de sa publication dans le journal (numéro spécial éditorialisé) « les limites de la liberté d'expression n'ont pas été dépassées ». Cette interprétation sera confirmée par la Cour d'appel de Paris en $2008^{41}$.

Ces décisions soulignent l'importance du contexte et la finalité poursuivie. La condition d'absence de «gratuité de l'offense », que l'on trouve dans la jurisprudence de la CEDH depuis l'arrêt Otto Preminger Institut est ici consacrée par l'existence d'un débat public auquel le journal se propose de contribuer. Mais la satire n'a-t-elle pas aussi pour objet de provoquer ce débat public ? On mentionnera pour mémoire la « réussite » médiatique de la campagne d'affichage «Unhate » de Benetton basée sur des photomontages, l'un d'eux montrant le pape embrassant le grand imam du Caire ${ }^{42}$.

Ces contentieux montrent en tout cas que la caricature n'est pas nécessairement traitée sous l'angle des lois du genre et notamment de la recherche de l'intention humoristique, même si elle n'en est pas nécessairement absente. Comme le soulignent fort bien les juges du fond, si certaines inspirent d'avantage la peur que le sourire, elles n'en doivent pas moins être tolérées pour autant.

On notera pour l'anecdote que le Président de la République d'alors, Nicolas Sarkozy, était venu exprimer son soutien à Charlie Hebdo à l'ouverture du procès en première instance. «Je préfère l'excès de caricatures à l'absence de caricature », avait-il écrit dans une lettre

\footnotetext{
${ }^{40}$ TGI Paris, $17^{\mathrm{e}}$ ch. corr., 22 mars 2007 : Recueil Dalloz, 2007, p. 1023 ; RSC 2007, p. 564, obs. Jacques Francillon ; Dr. pénal, 2007, Comm. ${ }^{\circ} 66$, note Agathe Lepage ; JCP 2007. II. 10079, note Emmanuel Derieux.

${ }^{41}$ Paris, $11^{\mathrm{e}}$ ch. A, 12 mars 2008, Légipresse n' ${ }^{\circ}$ 252. III. 107, note Henri Leclerc; Recueil Dalloz, 2009, p. 1779 obs. JeanYves Dupeux et Thierry Massis.

${ }^{42}$ Voir déjà en 1992, la campagne « Kissing Nun ».
} 
adressée au directeur de Charlie Hebdo, Philippe Val, lue à l'audience par l'avocat de ce dernier. Pouvait-il se douter que moins d'un an plus tard, il se retrouverait lui-même sur le banc des victimes d'une caricature?

\section{L'image épinglée, caricature et droits de la personnalité}

La protection juridique des droits de la personnalité, de l'honneur à la vie privée jusqu'au droit à l'image (C. civ. art. 9) est trop riche en débats théoriques et en décisions jurisprudentielles pour pouvoir être approfondie dans le cadre de nos développements. Sans trop s'étendre, on remarquera toutefois que le législateur n'a pas jugé bon de mentionner expressément la caricature, le pastiche ou la parodie comme exceptions à ces droits fondamentaux de la personne. C'est la jurisprudence qui les ajoutera, les rangeant en apparence sous la bannière de la liberté d'expression ${ }^{43}$ tout en rapprochant leur régime de la caricature applicable en droits d'auteur avec la référence aux «lois du genre ${ }^{44} »$. C'est en ce sens que se prononce par exemple la Cour de cassation en 1998 à propos d'«épinglettes» parlantes (ou pin's) réalisées à l'effigie de deux commentateurs sportifs : «chacun a le droit de s'opposer à la reproduction de son image $[\ldots]$ cette reproduction, sous forme de caricature n'est licite, selon les lois du genre, que pour assurer le plein exercice de la liberté d'expression ${ }^{45}{ }^{2}$.

Parce que la base textuelle fait défaut, une grande partie de la doctrine et de la jurisprudence considère qu'il s'agit d'une tolérance ${ }^{46}$, soulignant ainsi, mais en creux, ce qu'elle a d'incompatible avec l'expression d'une liberté. « La caricature constitue une tolérance traditionnellement admise à l'égard de ceux dont la profession ou l'activité permet de présumer de leur part une autorisation tacite ${ }^{47} »$.

La différence entre une tolérance et une liberté est fondamentale si l'on prend le temps d'y réfléchir. La manifestation d'une liberté n'a pas à être justifiée pour chaque espèce, elle doit seulement répondre de ses expressions abusives. Il appartiendra donc à la victime parodiée de démontrer en quoi les limites de cette liberté ont été franchies. Au contraire, la tolérance est par nature précaire, et tient au bon vouloir de l'intéressé. Le personnage parodié, caricaturé, peut alors permettre ou interdire.

\footnotetext{
${ }^{43}$ Paris, $1^{\mathrm{e}}$ ch., 11 mars 1991, Rainier III c/ Canal+ : Cah. dr. auteur 1991, n³7, p. 17.

${ }^{44}$ Voir en faveur d'un régime unitaire, Fiechter-Boulvard, op. cit., p. 67.

${ }^{45}$ Civ. 1re, 13 janv. $1998, n^{\circ} 95-13694$ : Bull. civ. I, n 14.

${ }^{46}$ Par ex. Bertrand, op. cit.

${ }^{47}$ Versailles, $1^{\mathrm{e}}$ ch., 31 janv. 1991, EDI 7 c/ Belmondo : Recueil Dalloz, 1991, IR, 182.
} 
Deux décisions illustrent la complexité des rapports entre la caricature et les droits de la personnalité à propos d'une même affaire, qui concerne la commercialisation d'une poupée vaudou à l'effigie du président de la République d'alors, M. Nicolas Sarkozy.

\section{Affaire Nicolas S. c/ Poupée Vaudou : acte I}

Le litige naît à partir de la commercialisation d'un coffret composé d'un livre et d'une poupée, et portant le titre « Nicolas Sarkozy, le manuel vaudou», attribué à Yael Rolognese. Sur chacune des faces du coffret est représentée l'image du président, en noir sur fond bleu, piquée de trois aiguilles. La poupée et les aiguilles sont offertes par l'éditeur.

Le plaignant demande le retrait de la vente du coffret. Il soutien que l'utilisation de son image sans son autorisation et malgré son opposition expresse « ne participe pas à la liberté d'expression, mais sert exclusivement de moyen de promotion commerciale pour la vente d'un ouvrage $»$.

En référé, le juge du TGI de Paris ${ }^{48}$ refusera de donner satisfaction au demandeur. Après avoir rappelé que, conformément aux textes français et internationaux toute personne, même notoirement connue, a «droit au respect de sa vie privée » et de son image, attribut de sa personnalité, il poursuit en affirmant que ces droits doivent se concilier avec le droit à la liberté d'expression auquel il rattache « la caricature et la satire, même délibérément provocantes ou grossières ». Admettant que « le droit à l'humour connait des limites, telles que les atteintes au respect de la dignité de la personne humaine, l'intention de nuire et les attaques personnelles », le juge rejette la mesure de retrait demandée qui serait, en l'espèce, une atteinte disproportionnée à la liberté d'expression.

La décision est tout à fait originale s'agissant de la reproduction de l'image d'une personne sous forme de caricature dans le cadre de la vente d'un produit commercial. En effet, selon une jurisprudence bien établie, le droit à la caricature n'en permet pas l'exercice « quel que soit le support utilisé et [...] le droit de la commercialiser ${ }^{49}{ }^{4}$. L'exploitation commerciale de l'image d'autrui entraîne habituellement la condamnation par les tribunaux ${ }^{50}$. C'est donc sur une subtile distinction entre œuvre de l'esprit et produit purement commercial que s'attarde la décision du juge des référés pour trancher en faveur de la première qualification. Mais la distinction est-elle dans la qualification des produits de toute façon commercialisés ou

\footnotetext{
${ }^{48}$ TGI Paris, réf. 29 octobre 2008, n08-58400 : Recueil Dalloz, 2008 p. 2870 et la note Camille Le Douaron ; RLDI 2008/43, $\mathrm{n}^{\circ} 1422$, obs. L. Costes.

${ }^{49}$ Civ. 1re, 13 janv. $1998, n^{\circ} 95-13694$, préc.

${ }^{50}$ Du bateau de Pompidou aux publicités de Ryanair montrant Carla Bruni et Nicolas Sarkozy, TGI Paris, réf., 5 févr. 2008 : RTD civ. 2008, p. 273, obs. Jean Hauser.
} 
dans la finalité de l'opération ? Comme l'a très bien montré un auteur, « la parodie, selon la destination qu'on lui reconnaît, peut être, tour à tour, soit objet commercial, relevant de la liberté du commerce et de l'industrie, soit satire sociale, relevant de la liberté d'expression ${ }^{51} »$.

\section{Affaire Nicolas S. c/ Poupée Vaudou : acte II et dénouement}

Les magistrats de la cour d'appel de Paris en jugeront tout autrement le 28 novembre 2008 : «l'incitation du lecteur à piquer la poupée jointe à l'ouvrage avec les aiguilles fournies, action que sous-tend l'idée d'un mal physique, serait-il symbolique, constitue une atteinte à la dignité de la personne M. S. ${ }^{52}{ }$.

Les juges estiment pourtant que

la mesure d'interdiction sollicitée n'est pas proportionnée et adéquate en ce qu'elle est une mesure spécialement attentatoire à la liberté d'expression qui se manifeste dans cet ouvrage dont tous les éléments ne sont pas en cause et en ce qu'elle porte atteinte à l'œuvre dont les auteurs n'ont pas été appelés dans l'instance.

La société éditrice du livret fut cependant sommée d'apposer sur ses coffrets la mention :

Il a été jugé que l'incitation du lecteur à piquer la poupée jointe à l'ouvrage avec les aiguilles fournies dans le coffret, action que sous-tend l'idée d'un mal physique, serait-il symbolique, constitue une atteinte à la dignité de la personne M. S.

La décision est étonnante par son ambivalence. Elle affirme que la poupée est une «atteinte à la dignité de la personne » de Nicolas Sarkozy sans prononcer pour autant l'interdiction de la vente du produit symbolisant cette atteinte. L'explication peut être recherchée dans l'approche de l'affaire centrée sur la liberté d'expression, et non les lois d'un genre imprécis, dont les atteintes doivent être justifiées et surtout proportionnées. C'est ce que pourrait confirmer les motifs de la Cour choisissant la mesure lui paraissant la plus «adéquate » et «proportionnée » en l'espèce la mention de la décision judiciaire sur le coffret.

La conséquence de cette décision n'en aura pas été moins étonnante, sans être tout à fait surprenante. Le public se serait arraché le coffret « Nicolas Sarkozy le manuel vaudou » ainsi paré du bandeau judiciaire. Au total, il en aurait été vendu 4 fois plus ${ }^{53}$ que de coffrets «Ségolène Royal le manuel vaudou ». La Cour d'appel n'avait-elle pas garanti l'efficacité de la poupée ${ }^{54}$ ?

\footnotetext{
${ }^{51}$ Bernard Edelman, «Une belle victoire des Guignols de l'Info » : Recueil Dalloz, 1999, p. 449.

${ }^{52}$ Paris, $14^{\mathrm{e}}$ ch., 28 nov. 2008, Nicolas S. c/ Sté Tera Prod. ; Bernard Edelman, «Le Président piqué... dans sa dignité » : Recueil Dalloz, 2009, p. 610.

53 «Brèves », Legalis du mardi 16 déc. 2008, «Poupées vaudou à l'effigie de Nicolas Sarkozy : pas d'interdiction»: http://www.legalis.net/spip.php?page=breves-article\&id_article=2508 [consulté le 05/03/2014].

${ }^{54}$ V. le très savoureux dialogue imaginé par Bernard Edelman entre le libraire-sorcier et l'acheteur-vaudou : art. préc.
} 
Ces quelques décisions de justice ont toutes en commun de révéler un affranchissement progressif de la caricature, pastiche et parodie, des lois du genre dans lesquelles elles se trouvent parfois trop étroitement enchâssées. Ces détournements, au sens large, font partie des ressorts de la liberté d'expression, liberté qui, si l'on suit la jurisprudence de la Cour européenne des droits de l'homme, étend son domaine aussi bien à l'information, qu'à la création artistique ou à la publicité commerciale. Les limites de ces provocations, que la caricature incite au rire, provoque l'indignation ou invite à la réflexion et au débat, ne sont pas bornées par l'intention comique ou l'absence de confusion, mais par les limites plus larges qui sont celles de la liberté d'expression. Rappelons d'ailleurs, s'il en était besoin, le niveau élevé de protection du droit à la liberté d'expression lorsque les propos litigieux s'inscrivent « dans un débat d'intérêt général et relèvent de l'expression politique et militante ${ }^{55}$ ». L'intérêt de placer la discussion juridique sur ce terrain réside dans l'inversion de la méthode qu'elle permet. La satire ne doit pas se justifier, c'est une liberté. C'est l'atteinte à cette liberté qui doit, pour reprendre les conditions générales d'une ingérence dans un droit garanti, être prévue par la loi, poursuivre un but légitime et être nécessaire dans une société démocratique ${ }^{56}$.

\footnotetext{
${ }^{55}$ Parmi de nombreux arrêts, CEDH 25 févr. 2010, Renaud c/ France, ${ }^{\circ}$ 13290/07.

${ }^{56}$ Sur l'ensemble, Frédéric Sudre, Droit européen et international des droits de l'homme, PUF, 2005, p.208 sv.
} 